\title{
Left Ventricular Remodeling after Acute Myocardial Infarction and Biomarkers
}

Diana Zaliaduonyte-Peksiene and Justina Portacenko*

Department of Cardiology, Lithuanian University of Health Sciences, Kaunas, Lithuania

\begin{abstract}
Considering to pathogenetic mechanisms of $\mathrm{Ml}$ a few classical biomarkers have been used for diagnosis of $\mathrm{MI}$ or acute coronary syndrome. Lately novel markers have been identified to provide diagnostic and prognostic information and outcome of $\mathrm{Ml}$ or acute coronary syndrome. A few novel biomarkers are overviewed in this article. A few novel biomarkers mentioned in this article showed significant relation to left ventricle remodelling and poor prognosis after myocardial infarction.
\end{abstract}

Keywords: Left ventricular remodeling; New biomarkers; Acute myocardial infarction

\section{Introduction}

Myocardial infarction (MI) occurs as a result of coronary artery occlusion that obstructs the blood supply to the myocardium in the left ventricle (LV) [1]. LV remodeling can be described as cellular, interstitial, molecular and genetic changes that manifest clinically. These changes adjusts size, shape and function of the LV after a cardiac injury such as a myocardial infarction [2]. Myocardial infarction results in the migration of macrophages, monocytes, and neutrophils into the infarct zone; this initiate intracellular signaling and neurohormonal activation, which localizes the inflammatory response. Changes in circulatory system are determined by the loss of myocytes, the excitation of the sympathetic nervous system, actuation of renin-angiotensinaldosterone system or release of natriuretic peptides [3].

\section{Literature Review}

LV remodeling after MI has been divided into two phases: an early phase (via 72 hours) and a late phase (under 72 hours). In early remodeling phase infarct expansion results from the degradation of the intermyocyte collagen struts by serine proteases and the activation of matrix metalloproteinases (MMPs) released from neutrophils. Wall stress stimulates mechanoreceptors and transduces intracellular signals via angiotensin II (Ang II) release, which presents the increased synthesis of contractile assembly units. Disturbation in circulatory hemodynamics trigger the sympathetic adrenergic system, which stimulates catecholamine, activates the renin-angiotensin-aldosterone system, and induces the production of atrial and brain natriuretic peptides (ANP, BNP). Late remodeling phase involves myocyte hypertrophy and changes in ventricular structure to distribute the increased wall stresses. In this phase myocyte hypertrophy is initiated by neurohormonal and renin-angiotensin system activation, myocardial extension, and paracrine-autocrine factors $[3,4]$.

\section{Remodeling of $\mathrm{LV}$ develops in few different mechanisms}

1. Inflammation (C-reactive protein, TNF- $\alpha$, soluble TNF receptors, Fas, interleukins (I, 6 and 18), osteoprotegerin, adiponectin)

2. Hypertrophy/fibrosis (Matrix metalloproteinases, collagen propeptides, galectin-3, soluble ST2).

3. Apoptosis (GDF-15)

4. Misc (MicroRNA, quiescin Q6, VEGFR-1)

Considering to pathogenetic mechanisms of MI a few classical biomarkers have been used for diagnosis of MI or acute coronary syndrome. Lately novel markers have been identified to provide diagnostic and prognostic information and outcome of MI or acute coronary syndrome.

\section{Classical Biomarkers}

Three biomarkers assess different pathways implicated in the pathogenesis of LV remodeling:

1. Troponin $\mathrm{I}(\mathrm{TnI})$ increase indicates myocyte injury

2. Brain (B-type) natriuretic peptide (BNP) is increased in response to LV overload

\section{C-reactive protein (CRP) is a marker of inflammation}

\section{Troponin I (TnI)}

Troponin I is a part of the troponin complex. The troponin complex consists of three subunits that regulate the calcium-mediated contractile process of striated muscle. These include troponin C, which binds $\mathrm{Ca}^{+}$, troponin I (TnI), which binds to actin and inhibits actin-myosin interactions, and troponin $\mathrm{T}(\mathrm{TnT})$, which binds to tropomyosin, thereby attaching the troponin complex to the thin filament. Following myocyte injury, the initial release of cTnT and cTnI is from the cytosolic pool, followed subsequently by release from the structural (myofilamentbound) pool [5].

For MI, cardiac troponin $\mathrm{T}$ (cTnT) and cardiac troponin I (cTnI) are regarded as more sensitive and specific than other cardiac biomarkers. cTnT and cTnI remain in the blood stream approximately more than 10 days, reaches to peak approximately 1 day to 2 days after myocardial injury as suggested by Thygesen and Alpert in 2000. Because of its prolonged release in the blood, these biomarkers are useful diagnosing sub-acute myocardial infarction [4]. Studies showed that patients with elevated admission $\mathrm{cTnI}$ had a significantly higher incidence of cardiac mortality and of cardiac events, i.e., death, nonfatal myocardial infarction, target-vessel revascularization [6].

*Corresponding author: Justina Portacenko, Department of Cardiology, Lithuanian University of Health Sciences, Kaunas, Lithuania, Tel no: +37037 327201; E-mail: justuke1988@gmail.com

Received June 01, 2017; Accepted August 07, 2017; Published August 10, 2017

Citation: Zaliaduonyte-Peksiene D, Portacenko J (2017) Left Ventricular Remodeling after Acute Myocardial Infarction and Biomarkers. J Cardiovasc Dis Diagn 5: 291. doi: 10.4172/2329-9517.1000291

Copyright: (C 2017 Zaliaduonyte-Peksiene D, et al. This is an open-access article distributed under the terms of the Creative Commons Attribution License, which permits unrestricted use, distribution, and reproduction in any medium, provided the original author and source are credited. 


\section{Natriuretic peptides (bnp and nt-probnp)}

Brain (B-type) natriuretic peptide (BNP) is a 32-amino acid peptide that is synthesized and released predominantly from ventricular myocardium in response to myocyte stretch. Like atrial natriuretic peptide (ANP), BNP seems to have almost exclusively beneficial physiological properties, including balanced vasodilation, natriuresis, and inhibition of both the sympathetic nervous system and the reninangiotensin-aldosterone axis [7]. It is synthesized in ventricular myocardium and is released in response to increased wall stress [4]

In DETECT study were shown that elevated NT-pro-BNP plasma levels at baseline were associated with increased 5 -year hazard ratios for all-cause mortality (HR 5.02; CI 3.26-7.72; $\mathrm{p}<0.0001$ ) and for incident major cardiovascular events (HR 4.38; CI 2.82-6.80; $\mathrm{p}<0.0001$ ), consisting of cardiovascular events and mortality due to myocardial infarction or sudden cardiac death [8].

\section{C-reactive protein $(\mathrm{CRP})$}

C-reactive protein (CRP) is an acute inflammatory phase protein which activates macrophages and oxidative stress. It is considered as a risk factor and biomarker for cardiovascular disease. Increased level of CRP is associated with left ventricular (LV) dysfunction and demonstrate higher mortality and morbidity risk in patients with heart failure. It is an independent factor of ischemic or non-ischemic etiology and other cardiovascular risk factors [9]. CRP is reported to promote fibrosis and inflammation in angiotensin II induced myocardial remodeling [10].

Study by Orn et al. showed that two days following PCI, CRP levels were strongly correlated to NT-proBNP level, infarct size and LVEF. There was no correlated to LV volumes, but was inverse correlation to non-infarcted LV mass. A similar pattern of correlations was seen at 1 week, but not after 2 months. In contrast to C-reactive protein, IL-6 was correlated with infarct size only at 2 days as assessed by CMR $(0.38$, $\mathrm{P}=0.01$ ), but not by enzymatic methods [11].

In the study by Schoos MM et al. were indicated that CRP at baseline was associated with infarct size, LVEF and end systolic volume (ESV) at baseline and 6 months follow up [12].

These findings suggest that CRP itself may play some role in the development of LV dysfunction and remodeling [9].

In addition, new studies by Hofmann et al. and Savvatis et al., showed that inflammatory factors deficiency, i.e., defect of IL-13 or IL23 , cause adverse remodeling after MI and can worsen outcomes after AMI [13,14].

\section{Discussion}

\section{Novel markers}

Matrix metalloproteinases (MMPs): Matrix metalloproteinases (MMPs) are a large family of calcium dependent and zinc containing endopeptidases. MMPs innitiate tissue remodeling and degradation of the extracellular matrix (ECM) such as collagen, elastins, matrix glycoproteins, proteoglycan, gelatin. They are controlled by hormones, growth factors and cytokines and are excreted by a variety of tissue and pro-inflammatory cells, i.e., fibroblasts, osteoblasts, endothelial cells, macrophages, neutrophils, lymphocytes [15]. So far 28 types of human matrix metalloproteinases have been recognized and been classified as collagenases, gelatinases, stromelysins, matrilysins, membraneType [16]. Researches of MI have showed increased levels of several MMPs. Most of all, MMPs-1, $-2,-3,-7,-8,-9,-13$, and -14 levels were increased. MMPs $-2,-8,-9$, and-13 have been evaluated in plasma and tissue samples [17]

MicroRNAs (miRNAs): MicroRNAs (miRNAs) are endogenously expressed small non-coding RNA molecules. Genes encoding miRNAs can be found in genomes of almost all organisms, including viruses. Their prime mechanism of action is posttranscriptional repression of gene expression [18]. It is suggested that the short length (22 nt) maximizes target gene specificity and minimizes non-specific effects. It is estimated that miRNAs regulate approximately $30 \%$ of genes within the human genome [19]. There are counted over 2000 encoded human miRNAs.

Most miRNA species are very stable and detectable in the peripheral blood or plasma, and the levels of circulating miRNAs varies in individuals with various pathological conditions, that make them excellent diagnostic and prognostic biomarkers for different diseases, i.e., cancer or diabetes $[20,21]$. In recent years, studies have reported the diagnostic value of miRNAs in the setting of AMI $[22,23]$. And new reports have examined the association among predictive value of circulating miRNAs and cardiac remodeling after AMI.

Pin et al. study revealed that plasma miR-208b and miR-34a could serve as available predictors for LV remodeling after AMI and were associated with the six months mortality or development of heart failure-23.1\% experienced this primary endpoint. MiR-208b is characterized as cardiac-specific microRNA in early diagnosis of AMI and where showed a correlation between plasma miR-208b and LV dysfunction after MI. Despite miR-34 family has protective attribute against pathological cardiac remodeling, though overexpression of miR-34a demonstrates induced endothelial cells aging and atherosclerosis [24].

In a study by Devaux et al. [25], low levels of miR-150 are associated with LV remodeling after the first ST-AMI. According to Devaux et al. [26], low levels of miR-150, miR-101 or elevated levels of miR-16 and miR-27a were at increased risk of impaired LV contractility and were involved in cardiac remodeling after MI.

Even so, two studies have shown that some of miRNAs (i.e., miR$133 \mathrm{a}$ and miR-423-5p) are not useful as biomarkers for prognosis of patients with STEMI or for an incidence of LV remodeling 1 year after anterior AMI [27,28].

Galectin-3: Galectin-3 is a $\beta$-galactoside-binding lectin secreted by activated macrophages, which has gained interest as at least a marker of, or possibly even a potential mediator in inflammation and fibrosis, processes that are central to the pathophysiology of LV remodeling $[29,30]$.

Tsai et al. suggested few important clinical implications of galectine-3: 1) circulating galectine-3 was significantly higher in AMI patients than in normal controls; 2) there were significant positive correlations of high circulating galectine- 3 to an advanced Killip score, unstable hemodynamics requiring IABP support, advanced CHF and a high CADILLAC risk score; 3 ) elevated galectin-3 was proven to be a strong independent predictor of 30-day MACO (major adverse clinical outcome) among patients with STEMI undergoing primary PCI [31].

Though, Weir et al. had demonstrated that higher galectin-3 concentrations at baseline were significantly associated with lower LVEF at 24-week follow-up, although there was no significant relationship between galectin-3 and remodeling per se [32].

Growth differentiation factor 15 (GDF-15): Growth differentiation factor 15 (GDF-15) is a protein that is encoded by this 
gene which belongs to the transforming growth factor-beta (TGF-beta) family. This protein is expressed in cells as a response in cell injury and acts pleiotropicaly. Increased protein levels are associated with tissue inflammation, hypoxia, acute cell injury or oxidative stress. In normal tissue condition, GDF-15 is not expressed however, during 'stress', its levels have been shown to increase [33].

\section{Conclusion}

A few clinical investigations showed that GDF-15 is an independent predictor of LV remodeling, death and heart failure in patients with STEMI. Echocardiographic and GDF-15 measurements were performed at baseline and were repeated 6 months or 12 months later. Studies demonstrated-patients with higher GDF-15 levels above the median had lower LV ejection fraction, increased risk of HF or death [34-36].

\section{References}

1. Jourdan-Lesaux C, Zhang J, Lindsey ML (2010) Extracellular matrix roles during cardiac repair. Life Sci 87: 391-400.

2. Cohn JN, Ferrari R, Sharpe N (2000) Cardiac remodeling-concepts and clinical implications: A consensus paper from an international forum on cardiac remodeling. J Am Coll Cardiol 35: 569-582.

3. Sutton MG, Sharpe N (2000) Left ventricular remodeling after myocardial infarction. Circulation 101: 2981-2988.

4. Libby $P$ (2012) The vascular biology of atherosclerosis. From: ZipesDP, Libby $P$, Bonow RO, Braunwald E (eds). Braunwald's Herat Disaese. A textbook of cardiovascular medicine, (9th edn), Philadelphia: Elsevier Saunders, USA. pp. 1087-1110.

5. Fathil MF, Arshad MM, Gopinath SC, Hashim U, Adzhri R, et al. (2015) Diagnostics on acute myocardial infarction: Cardiac troponin biomarkers. Biosens Bioelectron 70: 209-220.

6. Matetzky S, Sharir T, Domingo M, Noc M, Chyu KY, et al. (2000) Elevated troponin I level on admission is associated with adverse outcome of primary angioplasty in acute myocardial infarction. Circulation. 102: 1611-1616.

7. De Lemos JA, Morrow DA (2002) Brain natriuretic peptide measurement in acute coronary syndromes. Circulation 106: 2868-2870.

8. Leistner DM, Klotsche J, Pieper L, Palm S, Stalla GK, et al. (2013) Prognostic value of NT-pro-BNP and hs-CRP for risk stratification in primary care: Results from the population-based DETECT study. Clin Res Cardiol 102: 259-268.

9. Nagai T, Anzai T, Kaneko H, Mano Y, Anzai A, et al. (2011) C-Reactive protein overexpression exacerbates pressure overload-induced cardiac remodeling through enhanced inflammatory response. Hypertension 57: 208-215.

10. Zhang R, Zhang YY, Huang XR, Wu Y, Chung AC, et al. (2010) C-reactive protein promotes cardiac fibrosis and inflammation in angiotensin II-induced hypertensive cardiac disease. Hypertension 55: 953-960.

11. Orn S, Manhenke C, Ueland T, Damås JK, Mollnes TE, et al. (2009) C-reactive protein, infarct size, microvascular obstruction, and left-ventricular remodelling following acute myocardial infarction. Eur Heart J 30: 1180-1186.

12. Schoos MM, Munthe-Fog L, Skjoedt MO, Ripa RS, Lønborg J, et al. (2013) Association between lectin complement pathway initiators, C-reactive protein and left ventricular remodeling in myocardial infarction-A magnetic resonance study. Mol Immunol 54: 408-414.

13. Hofmann U, Knorr S, Vogel B, Weirather J, Frey A, et al. (2014) Interleukin-13 deficiency aggravates healing and remodeling in male mice after experimental myocardial infarction. Circ Heart Fail 7: 822-830.

14. Savvatis K, Pappritz K, Becher PM, Lindner D, Zietsch C, et al. (2013) Interleukin-23 deficiency leads to impaired wound healing and adverse prognosis after myocardial infarction. Circ Heart Fail 7: 161-171.

15. Verma RP, Hansch C (2007) Matrix metalloproteinases (MMPs): Chemicalbiological functions and (Q) SARs. Bioorg Med Chem 15: 2223-2268.

16. Gupta SP, Patil VM (2012) Specificity of binding with matrix metalloproteinases. In Matrix Metalloproteinase Inhibitors Springer Basel. pp. 35-56.
17. Iyer RP, De Castro Brás LE, Jin YF, Lindsey ML (2014) Translating Koch's postulates to identify matrix metalloproteinase roles in postmyocardial infarction remodeling. Circ Res 114: 860-871.

18. Bartel DP (2004) MicroRNAs: Genomics, biogenesis, mechanism, and function. Cell 116: 281-297.

19. Pillai RS, Bhattacharyya SN, Filipowicz W (2007) Repression of protein synthesis by miRNAs: How many mechanisms? Trends Cell Biol 17: 118-126.

20. Chen X, Ba Y, Ma L, Cai X, Yin Y, et al. (2008) Characterization of microRNAs in serum: A novel class of biomarkers for diagnosis of cancer and other diseases. Cell Res 18: 997-1006.

21. Mitchell PS, Parkin RK, Kroh EM, Fritz BR, Wyman SK, et al. (2008) Circulating microRNAs as stable blood-based markers for cancer detection. Proc Nat Acad Sci 105: 10513-10518.

22. Devaux Y, Vausort M, Goretti E, Nazarov PV, Azuaje F, et al. (2012) Use of circulating microRNAs to diagnose acute myocardial infarction. Clin Chem 58: 559-567.

23. Xiao J, Shen B, Li J, Lv D, Zhao Y, et al. (2014) Serum microRNA-499 and microRNA-208a as biomarkers of acute myocardial infarction. Int $\mathrm{J}$ Clin Exp Med 7: 136.

24. Lv P, Zhou M, He J, Meng W, Ma X, et al. (2014) Circulating miR-208b and miR-34a are associated with left ventricular remodeling after acute myocardia infarction. Int J Mol Sci 15: 5774-5788.

25. Devaux Y, Vausort M, McCann GP, Zangrando J, Kelly D, et al. (2013) MicroRNA-150: A novel marker of left ventricular remodeling after acute myocardial infarction. Circ Cardiovasc Genet 6: 290-298.

26. Devaux Y, Vausort M, McCann GP, Kelly D, Collignon O, et al. (2013) A pane of 4 microRNAs facilitates the prediction of left ventricular contractility after acute myocardial infarction. PLoS One 8: e70644.

27. Eitel I, Adams V, Dieterich P, Fuernau G, De Waha S, et al. (2012) Relation of circulating MicroRNA-133a concentrations with myocardial damage and clinica prognosis in ST-elevation myocardial infarction. Am Heart J 164: 706-714.

28. Bauters C, Kumarswamy R, Holzmann A, Bretthauer J, Anker SD (2013) Circulating miR-133a and miR-423-5p fail as biomarkers for left ventricular remodeling after myocardial infarction. Int J Cardiol 168: 1837-1840.

29. Dumic J, Dabelic S, Flögel M (2006) Galectin-3: An open-ended story. Biochim Biophys Acta 1760: 616-635.

30. Yang RY, Rabinovich GA, Liu FT (2008) Galectins: Structure, function and therapeutic potential. Expert Rev Mol Med 10: e17.

31. Tsai TH, Sung PH, Chang LT, Sun CK, Yeh KH, et al. (2012) Value and level of galectin-3 in acute myocardial infarction patients undergoing primary percutaneous coronary intervention. J Atheroscler Thromb 19: 1073-1082.

32. Weir RA, Petrie CJ, Murphy CA, Clements S, Steedman T, et al. (2013) Galectin-3 and cardiac function in survivors of acute myocardial infarction. Circ Heart Fail 6: 492-498.

33. Khan SQ, Ng K, Dhillon O, Kelly D, Quinn P, et al. (2009) Growth differentiation factor-15 as a prognostic marker in patients with acute myocardial infarction. Eur Heart J 30: 1057-1065.

34. Lin JF, Wu S, Hsu SY, Yeh KH, Chou HH, et al. (2014) Growth-differentiation factor-15 and major cardiac events. Am J Med Sci 347: 305-311.

35. Dominguez-Rodriguez A, Abreu-Gonzalez P, Avanzas P (2011) Relation of growth-differentiation factor 15 to left ventricular remodeling in ST-segment elevation myocardial infarction. Am J Cardiol 108: 955-958.

36. Gaggin HK, Januzzi JL (2015) Cardiac biomarkers and heart failure. ACC 10. 